\title{
A SYNOPSIS OF THE GENUS ORCHIDOTYPUS (ORCHIDACEAE, VANDOIDEAE) IN COLOMBIA, WITH DESCRIPTION OF A NEW SPECIES
}

\section{Dariusz L. Szlachetko, Marta Kolanowska ${ }^{1} \&$ Katarzyna MystKowska}

\begin{abstract}
A synopsis of the genus Orchidotypus Kraenzl. in Colombia is presented. Morphological descriptions of all national representatives of the genus are provided, together with information about their habitat, ecology and general distribution, detailed illustrations of the studied plants, and an identification key for Colombian Orchidotypus species. A new species discovered during these studies is described and a new generic combination is proposed.
\end{abstract}

Key words: Andes, Neotropics, Orchidotypus, Pachyphyllum, taxonomy

Dariusz L. Szlachetko, Marta Kolanowska \& Katarzyna Mystkowska, Department of Plant Taxonomy and Nature Conservation, University of Gdańsk, Wita Stwosza 59, 80-308 Gdańsk, Poland; e-mail: martakolanowska@wp.pl

\section{INTRODUCTION}

Pachyphyllinae Pfitzer is one of numerous problematic orchid taxa, variously classified. Dressler (1981), followed by Chase et al. (2003), included it in Cymbidieae Pfitzer, Senghas (1995) included it in Oncidieae Pfitzer, and Szlachetko (1995) included it in Dichaeeae Pfitzer. Most often only two genera are included in this subtribe: Fernandezia Ruiz \& Pav. and Pachyphyllum Kunth. Some authors (Senghas 1995; Szlachetko \& Mytnik-Ejsmont 2009) recognized a third genus, Orchidotypus Kraenzl. Due to its very tiny habit and inconspicuous flowers, difficult to study in herbarium material, the genus remains the least-known member of the subtribe.

Orchidotypus was described in 1906 by Friedrich W. L. Kraenzlin based on Orchidotypus muscoides Kraenzl., but it has been considered a synonym of Pachyphyllum by most taxonomists (e.g., Schlechter 1915; Schweinfurth 1961; Dressler 1993; Atwood \& Mora de Retana 1999). Christenson (2008) recognized it as a section of Pachyphyllum. Based on multiple DNA sequences and the patterns of modification for different pollinators, Chase and Whitten (2011) suggested that

\footnotetext{
1 Corresponding author
}

Raycadenco Dodson, Pachyphyllum and Orchidotypus should be combined with Fernandezia despite their significant morphological differences. However, Orchidotypus is easily distinguished from other Pachyphyllinae taxa by its connate sepals which form a prominent tube (Senghas 1995). The lip is clawed, similar in shape to the petals, with a pair of rounded calli. The gynostemium is short, delicate, and bent back or gently arched. The column part is about twice as long as the anther, and broadly winged. The wings are thin and rather close to each other in front of the gynostemium. There is no column foot. The plants have two pollinia which are slightly dorsiventrally compressed, obovoid to clavate, and rather hard. The apical clinandrium is well-developed, forming a very high collar, entire or irregularly denticulate on the margin, which completely hides the anther. The stigma is elliptic and concave. The rostellum is short and its remnant is very shortly bilobate at the apex. The viscidium is single, relatively large and elliptic-obovate, thin and lamellate (Szlachetko \& Mytnik-Ejsmont 2009).

Orchidotypus species grow as epiphytes in Central America and the north of South America, 
from Costa Rica to Bolivia. Most of them form clumps in moss tussocks in Andean mountain forests at elevations from 2500 to $3600 \mathrm{~m}$ a.s.l. (Senghas 1995). Of the 16 Pachyphyllum s.l. species reported from Colombia (Ortiz Valdivieso \& Uribe Vélez 2007), four represent Orchidotypus: O. bryophytus (Schltr.) Senghas, O. hispidulus (Rchb.f.) Senghas, O. schultesii (L. O. Williams) Senghas and $O$. vaginatus (Schltr.) Senghas. Both Pachyphyllum bryophytum and $P$. vaginatum were described by Schlechter (1924) based on plants collected by Hopf. Unfortunately the type specimens of both species were destroyed during a fire in the herbarium in the Berlin-Dahlem Botanical Museum in 1943 and no duplicates of those collections have been located since then.

In this study we sought to determine the present diversity of Orchidotypus in Colombia and to clarify the taxonomic affinities of the species that occur there.

\section{MATERIAL AND METHODS}

This revision of Orchidotypus is based on an examination of herbarium specimens. We revised $c a 200$ specimens of Orchidotypus and the related taxa Pachyphyllum and Fernandezia. Dried herbarium specimens deposited in COL were examined according to standard procedures. Each studied specimen was photographed and the data from the label were taken. Leaf form was studied first. Perianth parts and ovary structure were examined under a stereoscopic microscope after softening the flowers in water.

The key for determination of the Orchidotypus in Colombia is dichotomous. Abbreviations of authors' names follow Brummitt and Powell (1992). Information on the labels was used to describe the habitat requirements, flowering time and elevation range of each species. The information about flowering time and altitudinal range are specified for the Colombian populations.

\section{RESULTS}

We confirmed the occurrence of three Orchidotypus species in Colombia. The record of $O$. vaginatus in Columbia is limited to the original description of the species, since no specimen of the type collection was found.

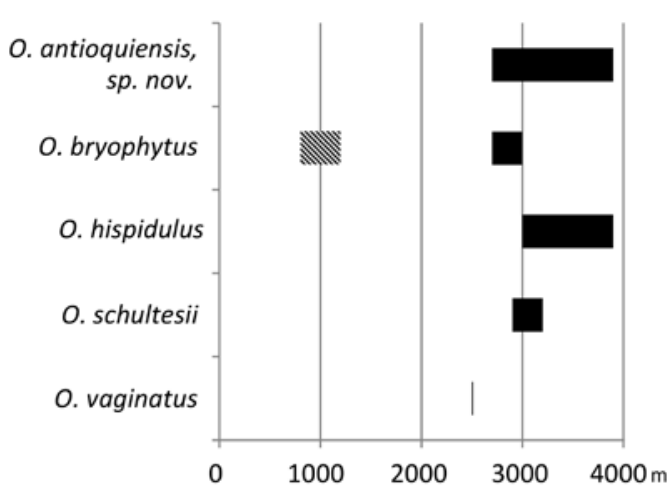

Fig. 1. Altitudinal range of Colombian Orchidotypus Kraenzl. species.

Colombian populations of Orchidotypus usually were found growing in paramo at 2700 $2900 \mathrm{~m}$ a.s.l. but their upper altitudinal limit extends to $3900 \mathrm{~m}$ a.s.l. A single population of O. bryophytus was found at the surprisingly low elevation of 800-1200 m a.s.l. but most probably this record is the result of incorrect collection data as we did not find any report of a Orchidotypus species growing in premontane areas in the whole range of the genus (Fig. 1).

This study revealed the existence of a distinctive Orchidotypus species which is described here as new. Additionally we propose a taxonomic transfer of one Peruvian species classified until now under Pachyphyllum.

The characteristics of all Colombian representatives of Orchidotypus are presented below. Information about the ecology of each species is provided and a map of the distribution of their populations is presented in Figure 2.

\section{TAXONOMIC TREATMENT}

Orchidotypus Kraenzl.

Bot. Jahrb. Syst. 37(4): 383. 1906.

GENERITYPE: Orchidotypus muscoides Kraenzl.

Small or tiny pendent epiphytes or terrestrials. Stems weak, branching only at base, concealed by leaf sheaths. Leaves fleshy, distichous, ciliatedentate on margins, blades falcate-lanceolate to elliptic, acute, diminishing in size apically, often 
twisted basally. Inflorescence axillary, 1-3-flowered, usually shorter than leaves. Flowers minute. Perianth parts connate into prominent tube. Lip similar to petals, with claw united to perianth tube, and a pair of rounded calli on each side above disc base. Gynostemium sessile, short, bent back or gently arched, delicate. Column part $c a$ twice as long as anther, broadly winged, wings thin, rather close to each other in front of gynostemium. Column foot absent. Anther ventral, incumbent, motile, dorsiventrally compressed, ellipsoid-cordate, obscurely 2-chambered. Connective narrow, thin. Pollinia 2, slightly dorsiventrally compressed, obovoid to clavate, rather hard. Caudiculae amorphous, sticky. Apical clinandrium well-developed, forming very high collar completely hiding anther, entire or irregularly denticulate on margin. Stigma elliptic, concave. Rostellum very short. Viscidium single, relatively large, elliptic-obovate, thin, lamellate. Tegula single, linear, very small, thin, lamellate. Rostellum remnant very shortly bilobate at apex.

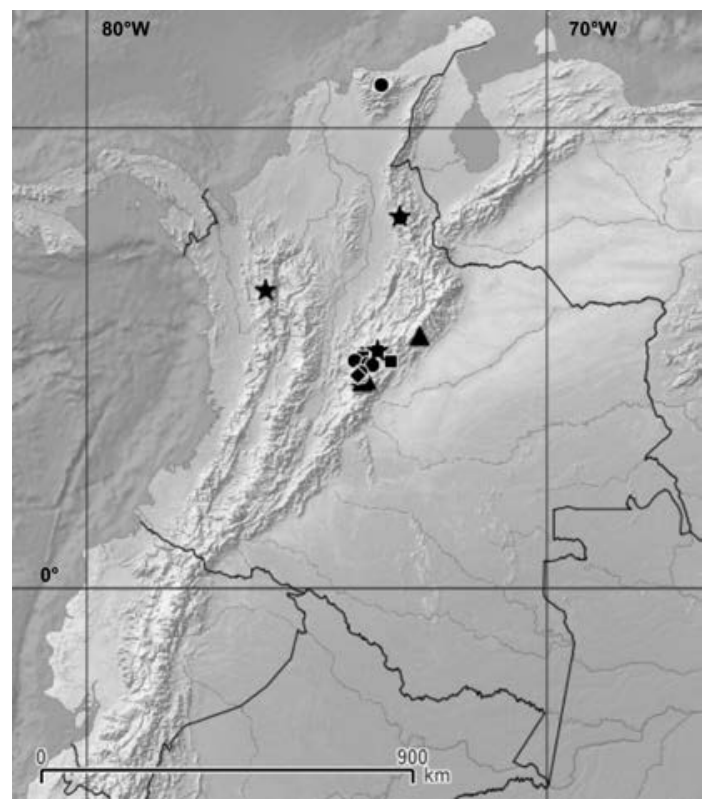

Fig. 2. Distribution of Orchidotypus species in Colombia: O. hispidulus (Rchb. f.) Senghas (•), O. bryophytus (Schltr.) Senghas ( $)$, O. antioquiensis Szlach., Kolan. \& Mystkowska,

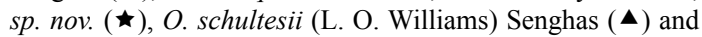
O. vaginatus (Schltr.) Senghas $(\bullet)$.

\section{Key to COLOMBIAN SPECIES OF ORCHIDOTYPUS}

1. Leaves completely glabrous ............ 2

1. Leaves ciliate, at least on their sheaths ..... . 3

2. Lip longer than petals $\ldots \ldots \ldots \ldots \ldots \ldots$

...... O. schultesii (L. O. Williams) Senghas

2. Lip subequal in length to petals ............

$\ldots \ldots \ldots \ldots$ O. vaginatus (Schltr.) Senghas

3. Leaf blade ciliate along margin ............

$\ldots \ldots \ldots$ O. hispidulus (Rchb. f.) Senghas

3. Leaf blade ciliate only in apical part ........ 4

4. Lip ovate-cordate ...................

$\ldots \ldots \ldots$ o. bryophytus (Schltr.) Senghas

4. Lip suborbicular-pentagonal . . . . . . . . . . .

O. antioquiensis Szlach., Kolan \& Mystkowska

Orchidotypus hispidulus (Rchb. f.) Senghas

Fig. 3

Orchideen (ed. 3) 31: 1923. 1995. - Aeranthes hispidulus Rchb. f., Linnaea 41: 31. ['1876'] 1877 - LECTOTYPE (here designated): Colombia, Santa Martha, Novae Granadae, Purdie s.n. (W!). - Campylocentrum hispidulum (Rchb. f.) Rolfe, Orchid Rev. 11: 246. 1903. - Pachyphyllum hispidulum (Rchb. f.) Garay \& Dunst., Venez. Orchid. Ill. 3: 236-237. 1965. - Fernandezia hispidula (Rchb. f.) M. W. Chase, Phytotaxa 20: 30. 2011.

Plants up to $6 \mathrm{~cm}$ tall, erect, ascending, occasionally branching in lower part, delicate, densely leafy. Leaves up to $1 \mathrm{~cm}$ long and $0.25 \mathrm{~cm}$ wide, oblong-elliptic to lanceolate-elliptic, attenuate towards subobtuse or subacute apex, falcate, somewhat twisted, conduplicate, rather thick, margins densely ciliate. Leaf sheaths obliquely ovate-triangular, ciliate on margins. Inflorescence few mm long, shorter than leaves, 2-3-flowered. Flowers inconspicuous, tiny. Floral bracts $1 \mathrm{~mm}$ long, ovate-triangular, acute. Ovary up to $1 \mathrm{~mm}$ long, almost sessile. Tepals thin, connate together in lower two/thirds, thickened on free part. Dorsal sepal $1.4-1.5 \mathrm{~mm}$ long in total, $0.5 \mathrm{~mm}$ wide, oblong, triangular in free apex, acute, 1-nerved. Petals $1.3-1.4 \mathrm{~mm}$ long, $0.5-0.6 \mathrm{~mm}$ wide, oblong, triangular-ovate in free apex, acute, 1-nerved. Lateral sepals $1.4-1.5 \mathrm{~mm}$ long, $0.5 \mathrm{~mm}$ wide, oblong, somewhat falcate, oblong-elliptic in free part, acuminate, 1-nerved. Lip 1.2-1.3 mm long in 
total, claw adnate to sepaline tube, free part $0.7-$ $0.8 \mathrm{~mm}$ wide when spread, suborbicular-elliptic, unconstricted, gently attenuate towards subacute apex; callus at base of lip lamina, consisting of two well-separated rhombic projections. Gynostemium $1.2 \mathrm{~mm}$ long, solid, apically truncate, clinandrium unlobed, very thick, fleshy.

ECOLOGY. Growing in paramo at 3000-3900 m a.s.l. Flowering occurs throughout the year. Outside Colombia, populations of this species have also been reported from cloud and high montane forest.

General distribution. Guatemala, Costa Rica, Panama, Colombia, Ecuador, Peru.

Representative SPeCimens: COLOMBIA. Cundinamarca. Mpio. Subachoque. Páramo de El Tablazo, 3300 m, 13 Oct. 2003, M. Hernández Schmidt, J. L. Fernández, M. Vélez, C. Agudelo \& Est. Biol. Veg.
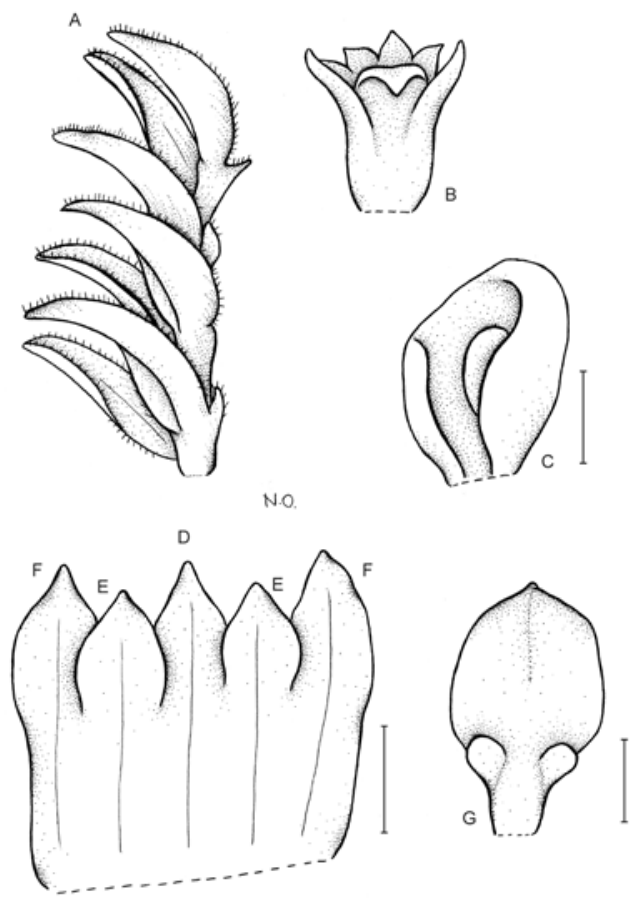

Fig. 3. Orchidotypus hispidulus (Rchb. f.) Senghas. A - leaf arrangement, B - flowers, C - gynostemium, D - dorsal sepal, E - petals, F - lateral sepal, G - lip. Scale bars $=0.5 \mathrm{~mm}$. Drawn by N. Olędrzyńska from Haught 5794 (COL!).
1377 (COL!); Road to E from Guasca, 3000 m, 31 May 1947, Haught 5794 (COL!); Carretera Guasca-Sueva, 3000 m, 22 Feb. 1977, M. Ospina H. 1476 (COL!); Bogotá, Páramo de Monserrate, vereda el Verjon, Hacienda Santa Barbara, El Granizo, 3000-3200 m, 9 July 1988, M. Garzón 562 (COL!); Quebrada del Chicó, Bogotá, 3000 m, 5 Sept. 1943, M. Schneider 105/1 (COL!); Magdalena, Sierra Nevada de Santa Marta, Valle de la Laguna Perdida, 3350 m, 30 Nov. 1977, O. Rangel 1396 (COL!); Sierra Nevada de Santa Marta, Aduriamana, 3400 m, 18 Aug. 1946, M. B. \& R. Foster \& E. Smith 1529 (COL!); Norte de Santander/Cesar, Limites entre los departamentos, Cordillera Oriental, Jurisdicciones, Cerro de Oroque, 3000-3900 m, 22-27 July 1974, H. García Barriga \& R. Jaramillo 20683 (COL!).

Notes. This species is easily distinguished from other Colombian Orchidotypus by its densely ciliate leaves.

\section{Orchidotypus bryophytus (Schltr.) Senghas}

Fig. 4

Orchideen I/B: 31. 1995. - Pachyphyllum bryophytum Schltr., Repert. Spec. Nov. Regni Veg. Beih. 27: 181. 1924 - Holotype: Colombia. Dept. Cundinamarca. Near Bogotá. Alt. 2500 m. 1921, H. Hopf s.n. (B destroyed). - Fernandezia bryophyta (Schltr.) M. W. Chase, Phytotaxa 20: 29. 2011.

Plants up to $3 \mathrm{~cm}$ tall, erect, ascending, delicate, rather densely leafy. Leaves up to $0.5 \mathrm{~cm}$ long and $0.17 \mathrm{~cm}$ wide, oblong-elliptic to linearoblanceolate, attenuate towards acuminate apex, somewhat twisted basally, conduplicate, rather thick, apical margins rather loosely ciliate. Leaf sheaths obliquely ovate-triangular, ciliate on margins. Inflorescence few $\mathrm{mm}$ long, somewhat longer than leaves, 2-3-flowered. Flowers inconspicuous, tiny. Floral bracts $1 \mathrm{~mm}$ long, ovatelanceolate, acuminate. Pedicel $0.8 \mathrm{~mm}$ long. Ovary up to $2 \mathrm{~mm}$ long. Tepals thin, connate together in lower half, thin, all acuminate. Dorsal sepal 2.6-2.7 mm long in total, $0.65-0.7 \mathrm{~mm}$ wide, oblong, oblong-lanceolate in free apex, 1-nerved. Petals 2.6-2.7 mm long, 0.5-0.6 mm wide, oblong, oblong-lanceolate in free apex, obliquely acute, 1-nerved. Lateral sepals 2.6-2.7 mm long, $0.6 \mathrm{~mm}$ wide, oblong, somewhat falcate, lanceolate in free part, 1-nerved. Lip 2.6-2.7 mm long 


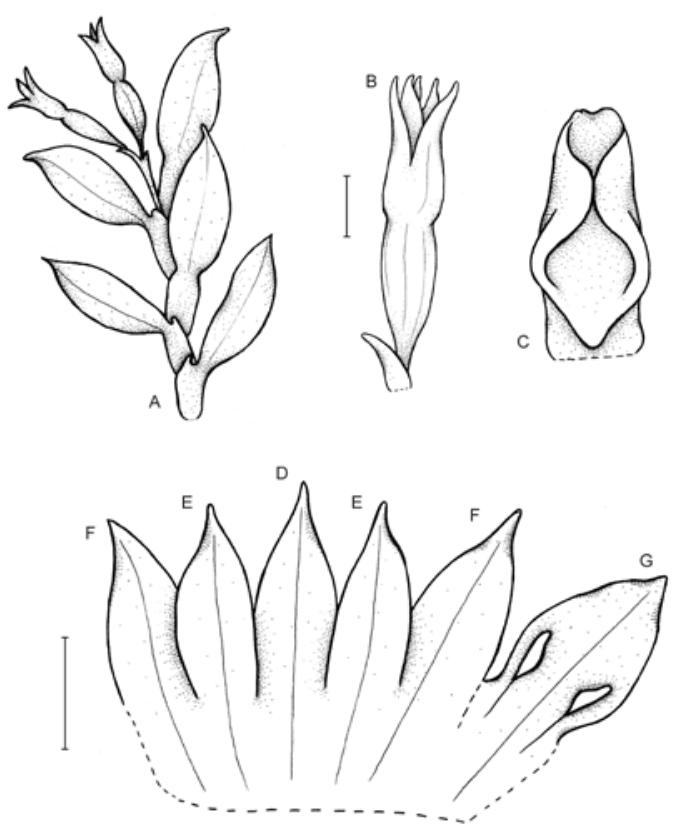

Fig. 4. Orchidotypus bryophytus (Schltr.) Senghas. A - plant habit, B - flower, C - clinandrium, D - dorsal sepal, E petals, F - lateral sepal, G - lip. Scale bars $=1 \mathrm{~mm}$. Drawn by N. Olędrzyńska from H. García Barriga 17209a (COL).

in total, claw adnate to sepaline tube, free part 1.1-1.2 mm long, 0.6-0.7 mm wide when spread, ovate-cordate, unconstricted, acute; callus at base of lip lamina, consisting of two well-separated wings. Gynostemium $1.8 \mathrm{~mm}$ long, solid, apically truncate, clinandrium unlobed, very thick, fleshy.

ECOLOGY. Growing in paramo at 2700-3000 m a.s.l. Flowering occurs throughout the year. We doubt the correctness of the locality data of H. García Barriga 17029a, cited below, as to our knowledge no Orchidotypus species have been found at such a low elevation.

General Distribution. Endemic to Colombia.

RePRESENTATIVE SPECIMENS: COLOMBIA. Boyacá, Carretera de Guateque a Santa Maria, Entre Santa Maria y Piedra Campana, 800-1200 m, 10-12 March 1960, H. García Barriga 17209a (COL!); Cundinamarca, Zipaquira, Pena de la Vieja, 3000 m, 8 Sept. 1942, G. Huertas \& L. Camargo s.n. (COL!); Zipaquira, cerca de Cataluna, 2850 m, 12 Oct. 1942, G. Huertas \& L. Camargo 1124 (COL!); Choconta, El Sisga, 2700-2800 m, 21 Feb. 1962, H. García Barriga 17422 (COL!).
Notes. According to Schlechter (1924), in lip form this species resembles Pachyphyllum pseudodichaea Rchb.f., but the habit and connate tepals of $O$. bryophytus clearly distinguish these two taxa.

Orchidotypus antioquiensis Szlach., Kolan \& Mystkowska, sp. nov.

Fig. 5

Species similar in habit to O. hispidulus (Rchb. f.) Senghas and O. schultesii (L. O. Williams) Senghas, distinguished by having ciliate leaf apices and sheath margins as well as by a suborbicularpentagonal lip.

Holotype: COLOMBIA. Antioquia, Mpio, Urrao, Páramo de Frontino, Sitio Llano Grande, 3420 m, 11 Sept. 1986, F. Roldán, O. Marulanda \& M. Escobar 390 (COL).

Plants 3-5 cm tall, erect, ascending, densely leafy. Leaves up to $0.7 \mathrm{~cm}$ long and $0.2 \mathrm{~cm}$ wide, lanceolate, attenuate towards acute apex, twisted basally, conduplicate, rather thick, apical margins minutely ciliate. Leaf sheaths obliquely ovate-triangular, ciliate on margins. Inflorescence $5 \mathrm{~mm}$ long, longer than leaves, 2-4-flowered. Flowers
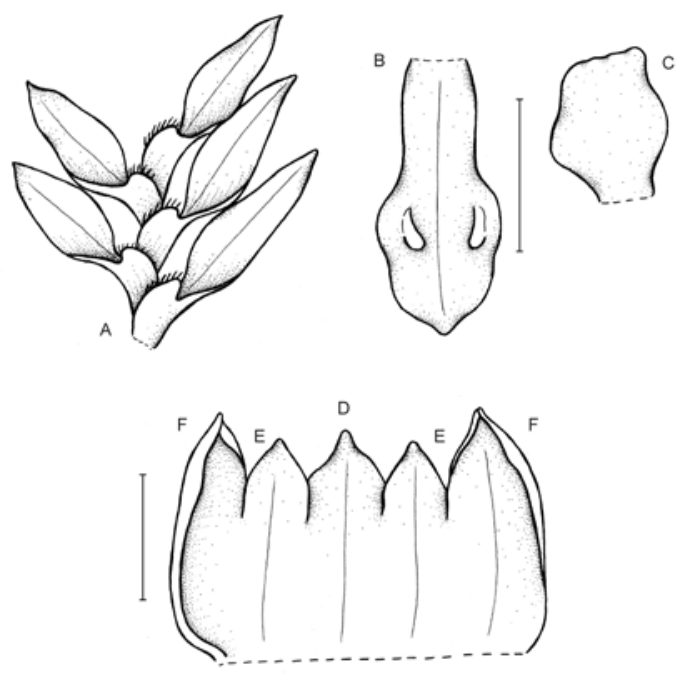

Fig. 5. Orchidotypus antioquiensis Szlach., Kolan. \& Mystkowska sp. nov. A - leaf arrangement, B - lip, C - clinandrium, $\mathrm{D}$ - dorsal sepal, $\mathrm{E}$ - petals, $\mathrm{F}$ - lateral sepals. Scale bars = $1 \mathrm{~mm}$. Drawn by N. Olędrzyńska from holotype. 
inconspicuous, tiny. Floral bracts $2 \mathrm{~mm}$ long, triangular, acute. Pedicel 1.0-1.2 mm long. Ovary up to $1.3 \mathrm{~mm}$ long. Tepals thin, connate together in lower two/thirds. Dorsal sepal 1.7-1.8 mm long in total, $0.6-0.7 \mathrm{~mm}$ wide, oblong, shortly acuminate at acute apex, 1-nerved. Petals $1.5 \mathrm{~mm}$ long, 0.6-0.7 $\mathrm{mm}$ wide, oblong, triangular-ovate in free apex, acute, 1-nerved. Lateral sepals 1.8$2.0 \mathrm{~mm}$ long, $0.7-0.8 \mathrm{~mm}$ wide, oblong-elliptic, somewhat falcate, ovate in free part, acuminate, 1-nerved, concave. Lip 1.6-1.8 mm long in total, claw adnate to sepaline tube, free part $1 \mathrm{~mm}$ long and $0.7-0.8 \mathrm{~mm}$ wide when spread, suborbicularpentagonal in outline, unconstricted, gently attenuate towards subobtuse apex; callus above base of lip lamina, consisting of two well-separated, obliquely oblong projections. Gynostemium $1 \mathrm{~mm}$ long, solid, apically truncate, clinandrium unlobed, thick, fleshy.

ETYMOLOGY. In reference to the place of origin of the type specimen.

ECOLOGY. Growing in paramo at 3400-3900 m a.s.l. and probably in high-montane forest at 2700 $2900 \mathrm{~m}$ a.s.l. Flowering occurs in January, May and September.

General Distribution. Known exclusively from the Colombian Andes (Central and Eastern Cordilleras).

RePRESENTATIVE SPECIMENS: COLOMBIA. Antioquia, Mpio, Urrao, Páramo de Frontino, Sitio Llano Grande, 3420 m, 11 Sept. 1986, F. Roldán, O. Marulanda \& M. Escobar 390 (COL!); Cundinamarca, Choconta, El Sisga, Parte alta de La Represa, 2700-2900 m, 14 Jan. 1962, H. García Barriga 17384 (COL!); Norte de Santander/Cesar, Linea divisoria entre los departamentos, $20 \mathrm{~km}$ al S de Abrego, Las Jurisdicciones (Cerro de Oroque), 3700-3960 m, 19-21 May 1969, H. García Barriga \& R. Jaramillo 19784 (COL!).

Notes. This species resembles $O$. hispidulus and $O$. schultesii, both reported from Colombia. From those species it differs in having leaves ciliate only in the apical part and along the sheath margin, which are also observed in Colombian $O$. bryophytus and, newly combined in this paper, Peruvian O. aurorae (D. E. Benn. \& Christenson)
Szlach., Kolan. \& Mystkowska. From the latter species $O$. antioquiensis is easily distinguished by its lip form, which is 3-lobed in O. aurorae, according to the illustration provided by Bennett and Christenson (1998). The petals, being distinctly shorter than lateral sepals, as well as the form of the projections on the lip disc of the new species, resemble those observed in $P$. schultesii. From the Colombian species $O$. bryophytus this species differs by it slip shape and petals distinctly shorter than the lateral sepals. Unlike the new species, $O$. vaginatus has glabrous leaves and its ovateelliptic lip is subequal in length to the petals.

Orchidotypus schultesii (L. O. Williams) Senghas Fig. 6

Orchideen I/B(31): 1923. 1995. - Pachyphyllum schultesii L.O. Williams, Caldasia 1(3): 15, pl. s.n. [p. 16]. 1941. - Lectotype (here designated): COLOMBIA. Páramo de Chipaque, SE of Bogotá, 3200 m, 25 Sept. 1941, R. E. Schultes 1020 (AMES).

Plants up to $5 \mathrm{~cm}$ tall, erect, ascending, densely leafy. Leaves up to $0.7 \mathrm{~cm}$ long and $0.18 \mathrm{~cm}$ wide, oblong-elliptic to lanceolate-elliptic, attenuate towards acute apex, falcate, strongly twisted, conduplicate, rather thick, margins glabrous. Leaf sheaths obliquely ovate-triangular, glabrous on margins. Inflorescence $c a 3 \mathrm{~mm}$ long, shorter than leaves, 2-3-flowered. Flowers inconspicuous, tiny. Floral bracts $1 \mathrm{~mm}$ long, ovate-triangular, acute. Pedicellate ovary up to $2 \mathrm{~mm}$ long, triquetrous. Tepals thin, connate together in lower two/thirds. Dorsal sepal up to $1.2 \mathrm{~mm}$ long in total, 0.45 $0.50 \mathrm{~mm}$ wide, oblong with triangular free apex, acute, 1-nerved. Petals $1 \mathrm{~mm}$ long, $0.45-0.50 \mathrm{~mm}$ wide, oblong with triangular-ovate apex, acute, 1-nerved. Lateral sepals 1.4-1.5 mm long, 0.45$0.50 \mathrm{~mm}$ wide, oblong, falcate, with ovate-lanceolate free part, acute, 1-nerved. Lip up to $1.3 \mathrm{~mm}$ long in total, $0.40-0.45 \mathrm{~mm}$ wide, claw adnate to sepaline tube, free part $0.5-0.6 \mathrm{~mm}$ wide when spread, ovate-elliptic, unconstricted, subobtuse at apex, emarginate; callus at base of lip lamina, consisting of two well-separated oblong projections. Gynostemium $0.7 \mathrm{~mm}$ long, solid, apically rounded, clinandrium unlobed, very thick, fleshy. 

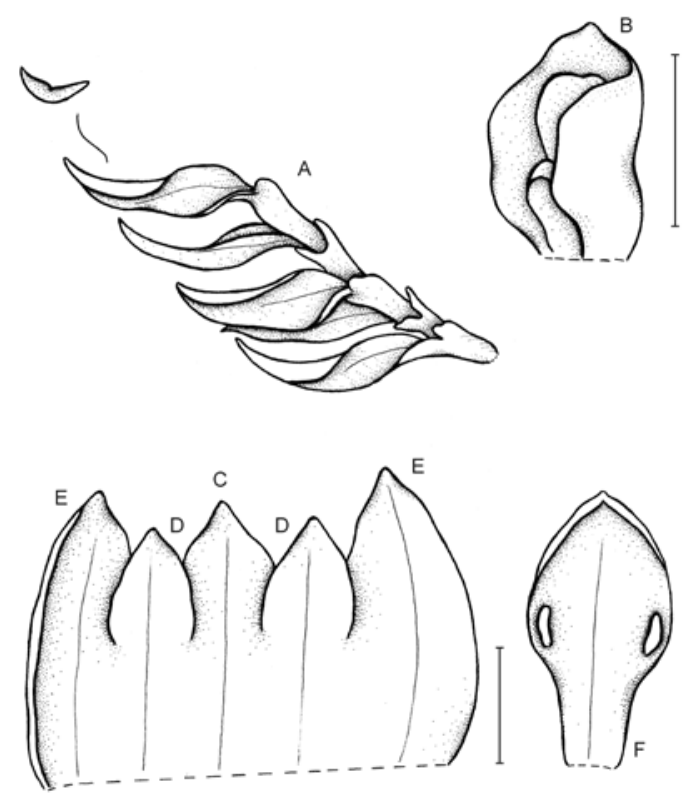

Fig. 6. Orchidotypus schultesii (L. O. Williams) Senghas. A - leaf arrangement, B - gynostemium, C - dorsal sepal, D - petals, E - lateral sepals, F - lip. Scale bars $=0.5 \mathrm{~mm}$. Drawn by N. Olędrzyńska from Schneider 272 (COL).

ECOLOGY. Growing in subparamo and paramo at 2900-3200 $\mathrm{m}$ a.s.1. Flowering occurs throughout the year.

General distribution. Colombia, Venezuela.

REPRESENTATIVE SPECIMENS: COLOMBIA. Boyacá, Vado Hondo, subparamo, Cnd. Jupal, $1500 \mathrm{~m}$ approx al NNE del caserio, 2900 m, 9 Apr. 1973, A. M. Cleef 9545 (COL!); Cundinamarca, Mpio. Choachi, Páramo de Cruz Verde, 25 May 1972, G. Lozano 2215 (COL!); Monserrate, 3200 m, 26 March 1944, M. Schneider 272 (COL!); Zipaquira, Pena de la Vieja, 3000 m, 8 Sept. 1942, L. Camargo \& D. Huertas s.n. (COL!).

\section{Orchidotypus vaginatus (Schltr.) Senghas}

Orchideen I/B: 31. 1995. - Pachyphyllum vaginatum Schltr., Repert. Spec. Nov. Regni Veg. Beih. 27: 182. 1924 - Holotype: COLOMBIA. Dept. Cundinamarca. Near Bogotá, Alt. 2500 m, 1921, H. Hopf s.n. (B destroyed). - Fernandezia vaginatum (Schltr.) M. W. Chase, Phytotaxa 20: 31. 2011.

Plant ca $5 \mathrm{~cm}$ tall, leafy. Leaves $0.50-0.75 \mathrm{~cm}$ long and $0.20-0.25 \mathrm{~cm}$ wide, lanceolate, thick, margins glabrous. Inflorescence shorter than leaves, few-flowered. Flowers inconspicuous, tiny. Floral bracts subequal in length to ovary, deltoid, acute. Pedicellate ovary ca $1 \mathrm{~mm}$ long, triquetrous-clavate. Tepals thin, connate together in lower half, forming a tube. Sepals $c a 1.75 \mathrm{~mm}$ long, oblong, obtuse, 1-veined. Petals slightly shorter than sepals, narrowly oblong. Lip subequal in length to petals, basally narrow, ligulate, ovate-elliptic, obtuse, with 2 short but distinctive projections above middle. Gynostemium $\mathrm{ca} 1 \mathrm{~mm}$ long, clinandrium subquadrate, apex truncate, with triangular apiculus.

\section{ECOLOGY. Growing at $2500 \mathrm{~m}$ a.s.1.}

General Distribution. Known exclusively from the locus classicus.

Notes. The characteristics given above follow Schlechter's original description of the species (Schlechter 1924). According to Schlechter, $O$. vaginatus resembles $O$. muscoides but differs from this species in both leaf and lip form. To our knowledge no specimen of $O$. vaginatus was found after Schlechter's description of the species. According to the characteristics given by the author it is distinguished by its glabrous leaves, petals shorter than sepals (subequal in length to the lip), and clawed, ovate-elliptic lip with two projections above the middle.

\section{NeW COMBINATION}

Orchidotypus aurorae (D. E. Benn. \& Christenson) Szlach., Kolan. \& Mystkowska, comb. nov.

Basionym: Pachyphyllum aurorae D. E. Benn. \& Christenson, J. Bot. Res. Inst. Texas 2: 288. 2008. - TyPE (Christenson 2008): Peru. Chanchamayo, alt. $2700 \mathrm{~m}$, 12 Apr. 1996, O. del Castillo ex Bennett 7501 (LECTOTYPE: MOL; ISOLECTOTYPE: NY).

ACKNOWLedGements. We thank the Curator and staff of the National Herbarium of Colombia for their kind hospitality and assistance during visits and for making specimens available on loan. We are grateful to the anonymous reviewers for helpful remarks on the manuscript and to Natalia Olędrzyńska for preparing the illustrations. The research described here was supported by 
the Polish Ministry of Science and Higher Education (research grant no. 8124/B/PO1/2011/40)

\section{REFERENCES}

Atwood J. T. \& Mora de Retana D. E. 1999. Orchidaceae: tribe Maxillarieae: subtribes Maxillariinae and Oncidiinae. Fieldiana, Bot. 40: 1-182.

Bennett D. E. \& Christenson E. A. 1998. Icones Orchidacearum Peruviarum. 3. A.Pastorelli de Bennett, Lima.

Brummitt R. K. \& Powell C. E. 1992. Authors of plant names: a list of authors of scientific names of plants, with recommended standard forms of their names, including abbreviations. Royal Botanic Gardens, Kew.

Chase M. W. \& WhitTen M. 2011. Further taxonomic transfers in Oncidiinae (Orchidaceae). Phytotaxa 20: 26-32.

Chase M. W., Cameron K. M., Barrett R. L. \& FreudenSTEIN J. V. 2003. DNA data and Orchidaceae systematics: a new phylogenetic classification. In: K. W. Dixon, S. P. Kell, R. L. Barrett \& P. J. CribB (eds), Orchid conservation, pp. 69-89. Natural History Publications, Kota Kinabalu.

Christenson E. A. 2008. A synopsis of Pachyphyllum (Orchidaceae). J. Bot. Res. Inst. Texas 2: 285-289.

DRESSLER R. L. 1981. The orchids: natural history and classification. Harvard University Press, Harvard.
DRESSLER R. L. 1993. Phylogeny and classification of the orchid family. Dioscorides Press, Portland.

KraENZLIn F. 1906. Orchidaceae andinae imprimis peruvianae Weberbauerianae. 3. Wilhelm Engelmann, Leipzig.

Ortiz Valdivieso P. \& Uribe Vélez C. 2007. Galería de Orquideas de Colombia (CD edition). Asociación Bogotana de Orquideología, Bogotá.

SCHLECHTER R. 1915. Die Orchideen, ihre Beschreibung, Kultur und Züchtung; Handbuch für Orchideen Liebhaber, Züchter und Botaniker. P. Parey, Berlin.

Schlechter R. 1924. Beitraege zur Orchideenkunde von Colombia, III. Orchidaceae novae vel rariores collectorum variorum. Repert. Spec. Nov. Regni Veg. Beih. 27: 1-183.

Schweinfurth C. 1961. Orchidaceae, Orchids of Peru. Fieldiana, Bot. 30(4): 787-1005.

Senghas K. 1995. 749. Chytroglossa-770. Sutrina. In: R. SCHLECHTER, Die Orchideen. Ed. 3. 1B(31): 1905-1976. Paul Parey, Berlin.

SzlachetKo D. L. 1995. Systema Orchidalium. Fragm. Florist. Geobot. Suppl. 3: 1-152.

Szlachetko D. L. \& Mytnik-Ejsmont J. 2009. Gynostemia Orchidalium IV. Orchidaceae-Vandoideae (Maxillarieae, Cyptarrheneae, Zygopetaleae, Dicheeae, Telipogoneae, Ornithocephaleae, Oncidieae). Acta Bot. Fenn. 180: 120-125.

Received 23 August 2014 